\title{
La innovación y sus efectos: la evidencia de los sectores manufactureros ecuatorianos y argentinos
}

\author{
Silvana Astudillo ${ }^{1 *}$ Anahí Briozzo ${ }^{2}$
}

Resumen: El objetivo del presente estudio consiste en comparar los efectos de la innovación en el producto y en el proceso en las micro, pequeñas y medianas empresas (MIPYMES) manufactureras argentinas y ecuatorianas. Se utilizó la base de datos de la Enterprise Survey del Banco Mundial (Panel 2006-2010) a través de un análisis multivariado y el control de la endogeneidad de la innovación. Los resultados evidencian que la innovación en el producto y en el proceso presentan efectos significativos diferenciales en ambos países.

Palabras Clave: innovación en el producto; innovación en el proceso; efectos; Argentina; Ecuador.

Abstract: The goal of this investigation is to compare the effects of the introduction of product and process innovation activities of micro, small and medium manufacturing enterprises (MSME), in respectively Argentina and Ecuador. For this purpose, World Bank's Enterprise Survey (Panel 2006-2010) data are used. A multivariate analysis was applied and included the innovation as an endogenous variable. The results show that the introduction of product and process innovations have significant differential effects in both countries.

Palabras Clave: product innovation; process innovation; effects; Argentina; Ecuador.

Submitted: July $17^{\text {th }} 2017$ / Approved: October $31^{\text {rd }} 2017$

\section{Introducción}

La introducción de innovaciones en los productos y en los procesos por las empresas manufactureras sugiere varias ventajas en sus resultados, sin embargo estas ventajas podrían ser diferentes entre empresas, industrias y entre países (Metcalfe, 1998). Así, la capacidad innovadora nacional de los países latinoamericanos ha mejorado su posición relativa en los últimos años y poseen diferentes indicadores de Ciencia Tecnología e Innovación (CTI) que hacen que experimenten impactos en su competitividad, aunque la inversión en este rubro es muy limitada.

Crespi y Dutrénit (2013) plantean que en el contexto latinoamericano, la mayoría de países han implementado similares enfoques de las políticas de CTI, pero con diferentes resultados. La comparación de los impactos de la innovación en diferentes economías ilustra la heterogeneidad y la diversidad de la región, así como las tendencias de convergencia y divergencia.

Por lo expuesto, la presente investigación tiene por objetivo realizar un estudio comparativo de los efectos de la innovación del producto $\mathrm{y}$ del proceso en las MIPYMES (micro, pequeñas, y medianas empresas) manufactureras argentinas y ecuatorianas. El Ecuador y la Argentina poseen diferente perfil en CTI, sin embargo presentan similares indicadores de desigualdad en la región. Esta investigación aporta con una nueva evidencia empírica a la comparabilidad de los efectos de innovación en economías latinoamericanas con diferente perfil de CTI especialmente en economías como el Ecuador en donde la evidencia empírica es escasa en lo que respecta a los impactos de la innovación. En la Argentina, existen investigaciones de los efectos de la innovación, sin embargo este trabajo se diferencia de los anteriores por el diseño metodológico y la base de datos.

A fines de analizar los efectos que generan las innovaciones en las empresas manufactureras ecuatorianas y argentinas, se utilizaron los datos a nivel de empresa con base a la Enterprise Survey del Banco Mundial en el periodo 2006-2010 a través de un análisis multivariado con un control endógeno de la innovación. La medición de los resultados de la innovación en las empresas manufactureras evidencia impactos por la introducción de las innovaciones en producto y en proceso, lo que sugiere enfocar a la innovación como una política pública para la mejora de la competitividad en las empresas de ambos países.

La estructura del artículo describe en la sección I el marco teórico y la literatura empírica, en la sección II se exponen los datos y la metodología, en la sección III se presenta los resultados y la discusión y la sección IV algunas conclusiones sobre los efectos de la innovación en cada país.

\section{Marco Teórico}

\subsection{Teoría de los efectos de la innovación}

En referencia a los efectos de la innovación, una aportación importante la realiza Schumpeter (1934), quien menciona los efectos positivos que entraña la generación de nuevos productos y procesos. Schumpeter define a la innovación como el desarrollo de un nuevo producto o proceso por parte de una empresa y su introducción exitosa en el mercado, el desarrollo de nuevas formas de organizar los negocios, nuevas fuentes de aprovisionamiento o la explotación de nuevos mercados.

(1) Universidad de Cuenca, Cuenca - Ecuador. Unidad de Innovación y Transferencia Tecnológica.

(2) Universidad Nacional del Sur, Bahía Blanca - Argentina. Departamento de Ciencias de la Administración IIESS (UNS-CONICET).

*Autor de correspondencia: silvana.astudillo@ucuenca.edu.ec 
Posterior al postulado de Schumpeter, la teórica evolucionista señala que el capitalismo está caracterizado por un proceso evolutivo asociado a la innovación. Una innovación significativa puede dar a una empresa una ventaja importante sobre otros (Nelson y Winter, 1982; Kline y Rosenberg, 1986). Malerba \& Orsenigo (1996) en un estudio en siete países sobre los patrones de innovación schumpeterianos explican que existen diferencias importantes como consecuencia de los efectos específicos de cada país en relación con los sistemas nacionales de innovación y las historias específicas de las empresas e industrias en cada país.

Posteriormente, la teoría endógena del crecimiento muestra la ventaja del enfoque schumpeteriano y su tratamiento explícito de la innovación como una actividad económica distinta, se introduce el paradigma de innovación endógena en el manejo de las políticas que influyen en el crecimiento (Grossman \& Helpman, 1993; Aghion, Howitt, Brant-Collett, \& García-Peñalosa, 1998).

Con Lundvall (1992) emerge el enfoque de los sistemas nacionales de innovación que sostiene que la innovación es un proceso sistémico con interacciones entre todos los actores de una nación. Con respecto a las ventajas comparativas de la innovación en los productos y en los procesos, Metcalfe (1998) establece que los resultados son diferentes para cada empresa por su desempeño innovador, no existen dos empresas que innoven de la misma manera ni a la misma velocidad. De hecho, Fagerberg (2004) concluye que los esfuerzos para promover la competitividad mediante la innovación rara vez pueden entenderse al margen de lo que otros están logrando al mismo tiempo. Lo que sugiere que el desempeño innovador es diferente en las firmas, industrias, regiones y entre países.

Lundvall (2009) considera que el motivo fundamental por el cual los gobiernos nacionales implementan políticas de innovación es el supuesto que la innovación es un elemento clave para el crecimiento económico nacional.

\subsection{Literatura empírica de los efectos de la innovación}

Los estudios empíricos manifiestan que la innovación genera un impacto positivo en las empresas (Crépon, Duguet, \& Mairesse, 1998; Lööf, Heshmati, Asplund, \& Nåas, 2001; Klomp \& Van Leeuwen, 2001; Sandven \& Smith, 2000; García Pérez De Lema \& Gálvez Albarracín, 2012, Gunday, Ulusoy, Kilic, \& Alpkan, 2011). Hassan, Shaukat, Nawaz, \& Naz (2013) sugieren la necesidad de una investigación comparativa de los efectos de la innovación cruzando las diferencias culturales. En esta línea Dutrénit \& Katz (2005) en un estudio de la innovación en Latinoamérica plantea necesario tener más conocimiento sobre la relación de los enlaces entre los esfuerzos de la innovación y resultados de innovación.

\section{Los inputs de la innovación y la performance empresarial}

Un aspecto que se presenta en la medición de los efectos de la innovación es la relación entre la innovación y sus determinantes y la relación de sus determinantes en los resultados de la empresa. Un primer aspecto que se analiza en las empresas son los factores que influyen en la decisión a innovar y luego como estos factores influyen la innovación a través de diferentes efectos. En el estudio comparativo sobre las determinantes de la innovación en el producto y en el proceso en las empresas manufactureras en la Argentina y en el Ecuador realizado por Astudillo y Briozzo (2015) se evidencian a la investigación y desarrollo, la calidad y los recursos humanos calificados.

En este sentido, autores como Griffith, Huergo, Mairesse, \& Peters (2006) señalan que existe una relación entre la investigación y desarrollo y la innovación y la productividad. Crépon, Duguet, \& Mairesse (1998) manifiestan que la investigación y el desarrollo constituyen una entrada de la innovación y la participación en ventas los resultados de la innovación. Lööf \& Heshmati (2002) manifiestan que el crecimiento de la productividad se correlaciona de manera positiva con los resultados de la innovación, incluso cuando se controla por el tamaño, la intensidad del uso de los factores y la destreza de la mano de obra. En este estudio, además del tamaño de la empresa se incluyen otras variables de control en el modelo con el fin de controlar los efectos de la innovación en la performance empresarial de las empresas que pudieran afectar el efecto de las variables independientes como la antigüedad, el género del gerente, el financiamiento externo y la competencia.

\section{Los outputs de la innovación y la performance empresarial}

El impacto de las innovaciones en las firmas sobre los resultados empresariales resulta ser variable para cada empresa. Por lo que en un esfuerzo de estandarizar definiciones sobre los efectos de la innovación se analizan algunos estudios que definen cuáles son los beneficios para los empresarios. En este sentido, el Manual de Oslo (OCDE, 2005, p.37) argumenta que es muy importante conocer porqué las empresas innovan:

Cuando se trata de innovaciones de proceso consiguen ganancias de productividad, la empresa se beneficia de una ventaja de costes sobres sus competidores. Cuando se trata de una innovación de producto, la empresa puede obtener una ventaja competitiva lanzando este nuevo producto, lo que le permite aumentar la demanda y su margen de beneficio.

Con base a estos indicadores, se presentan las variables que se persiguen en los objetivos del estudio y en la información provista en la Enterprise Survey del Banco Mundial. Se considera como un efecto importante el impacto económico de la innovación, existen algunas investigaciones que llegan a la conclusión que existen distintos objetivos perseguidos por las empresas como el incremento de las ventas, el aumento de la rentabilidad, el aumento de las exportaciones, la reducción de costos. En esta línea, Dosi et al. (1988), manifiestan que la innovación en el producto y en el proceso en las firmas o en las industrias implican diferentes oportunidades como beneficios económicos, aporte que concuerda con los resultados de Božić \& Radas, 2006, Evangelista \& Vezzani, 2010; Gómez \& Calvo, 2011; Reichert \& Zawislak, 2014. A este análisis, existe un cuestionamiento de Sandven \& Smith (2000) que mencionan una relación entre la rentabilidad y la innovación, pero no necesariamente en términos de ganancia. 
Con otro planteamiento, existen investigaciones en las industrias manufactureras, que señalan que la innovación en el producto tiene un enlace débil con las ventas de las empresas (Yeh-Yun \& Chen, 2007; Gunday, et al., 2011) . En referencia a las exportaciones, otros autores señalan que las pymes que tienen un historial de innovación son más propensos a exportar (Caldera, 2010; Martins, Goméz-Araujo, \& Vaillant, 2015; Love \& Roper, 2015). Por otra parte, las innovaciones permiten a las empresas reducir los costes marginales y obtener una ventaja sobre los competidores (Božić \& Radas, 2006; Caldera, 2010). En relación al impacto de la innovación en el mercado, la innovación puede ampliar el mercado e impulsar el crecimiento de la industria de manera positiva, en este argumento Drucker (1986) plantea que una innovación produce un cambio en el mercado o en la sociedad, lo que sugiere que las empresas pueden penetrar en nuevos mercados o tener una mayor cuota de mercado, esta afirmación la plantean también Lööf, et al., 2001; Klomp \& Van Leeuwen, 2001; Gómez \& Calvo, 2011; Brown \& Guzmán, 2014.

Con respecto a la relación de la innovación con el empleo, Pianta (2003) plantea que la innovación en el producto tiene un impacto positivo en el empleo y la innovación en el proceso con un efecto negativo. El efecto de la innovación de procesos en el empleo es negativo (Peters, 2008). Otro impacto de la innovación se presenta en la mejora de la calidad de los bienes y servicios y la obtención de certificaciones de calidad (Prajogo \& Sohal, 2003; Thai Hoang, Igel, \& Laosirihongthong, 2006). Los autores indican una relación positiva y significativa en particular de la innovación de procesos. Otro argumento de los efectos de la innovación como resultado de la actividad innovadora y analizada por el Banco Mundial es la utilización de menos materiales y energía por unidad producida, analizado por Gómez \& Calvo (2011) en la Encuesta sobre Innovación Tecnológica en las empresas del Instituto Nacional de Estadística de España en el año 2006.

En este marco, estudios realizados sobre los impactos en el desempeño de las firmas en el Ecuador son escasos, una primera aproximación sobre los objetivos para realizar las actividades de innovación se analizan en la primera encuesta de innovación 2009-2011 (SENESCYTINEC, 2014) que permite distinguir efectos como la mejora de la calidad de bienes o servicios, el aumento de la capacidad para producir bienes o servicios, el incremento de la participación de mercado, el aumento de la variedad de bienes o servicios, la mejora de la salud o seguridad ocupacional de sus empleados, el ingreso a nuevos mercados, la reducción de impactos ambientales, la reducción de costos.

En la Argentina, existen algunos estudios empíricos previos sobre los resultados de los esfuerzos de la innovación. Así por ejemplo, un estudio sobre la innovación y productividad en las compañías manufactureras argentinas en los años 90 realizado por Chudnovsky, Lopez, \& Pupato (2004) encuentra una relación entre el rendimiento de las empresas a través de una variedad de indicadores, como la mano de obra y la productividad total de los factores, los beneficios, las tasas de crecimiento de las ventas, los activos totales, las exportaciones a través de las determinantes como la investiga- ción y desarrollo y el tamaño. Planteamientos similares sobre los impactos de la innovación han sido considerados en los trabajos de Lugones, Suárez, \& Gregorini, 2007; Lugones, Suárez, \& Le Clech, 2007; Anlló \& Suárez 2009 que analizaron las encuestas de innovación elaboradas por el Instituto Nacional de Estadísticas y Censos- INDEC- de los períodos 1998, 2003, 2006 con la conclusión que la innovación genera un impacto positivo en las ventas, en las ganancias, en las exportaciones, en los salarios de los trabajadores y en sus características, además permite reducir los costos y aumenta la cuota de mercado.

En este sentido, con base a los argumentos anteriores se definen a continuación las siguientes hipótesis:

H1: La innovación en el producto genera impactos en la performance empresarial en ambos países tras controlar su endogeneidad

H2: La innovación en el proceso genera impactos en la performance empresarial en ambos países tras controlar su endogeneidad

\section{Metodología}

Para los propósitos de la investigación, una innovación de producto, se corresponde con la introducción de un bien o de un servicio nuevo significativamente mejorado, en cuanto a sus características o en cuanto al uso que se destina. Esta definición introduce la mejora significativa de las características técnicas, de los componentes y los materiales, de la informática integrada de la facilidad de uso u otras características funcionales. Una innovación de proceso es la introducción de un nuevo o significativamente mejorado, proceso de producción o de distribución que implica cambios significativos en las técnicas, los materiales, y/o programas informáticos (OCDE, 1995). En la Enterprise Survey del Banco Mundial las variables innovación en el producto y la innovación del proceso son medidas cualitativas resultados de la percepción de los empresarios. La base de datos denominada Panel 2006-2010 para cada país facilita los datos de los inputs de la innovación: investigación y desarrollo, calidad, recursos humanos calificados y los outputs de la innovación del año 2010, innovación en el producto y la innovación en el proceso. La performance empresarial (desempeño empresarial) en el año 2010 es medida como variación porcentual en las siguientes variables: ventas, exportaciones, productividad, empleo, fuerza laboral calificada, nuevos mercados, incremento de bienes ofrecidos, mejora de la calidad, reducción de costos y energía, obtención de certificaciones de calidad o de exportación. La separación temporal de las variables se realiza por la demora en la materialización de los efectos de la innovación y para evitar problemas de endogeneidad.

\subsection{Universo y Muestra}

El universo bajo estudio son las micro, pequeñas y medianas empresas manufactureras de la Argentina y Ecuador. A fines de este trabajo se consideran como MYPYMES solo aquellas empresas que contratan en forma permanente hasta 200 empleados. 
Se utiliza la base de datos de la Enterprise Survey del Banco Mundial del panel 2006-2010 ${ }^{1}$. El Banco Mundial toma una muestra estratificada siguiendo tres criterios: ubicación geográfica ${ }^{2}$, tamaño de la empresa según el número de empleados, y sector de actividad. La sub-muestra empleada en este estudio está compuesta por 54 MIPYMES manufactureras pertenecientes al Ecuador y 311 MIPYMES manufactureras de la Argentina.

En la Tabla 1, se muestra el número de empresas del sector manufacturero (MIPYMES) de la Argentina y el Ecuador. Se observa que el mayor número de empresas se concentran en la pequeña empresa de 10 a 49 empleados y la actividad más representativa en ambas naciones es la de Alimentos.

En la Argentina (Buenos Aires, Mendoza, Córdoba, Rosario) existen: 56 microempresas, 166 pequeñas empresas y 89 medianas empresas del sector manufacturero. En el Ecuador (Pichincha, Guayas, Azuay) se encuestaron a 10 microempresas, 28 pequeñas empresas y 16 medianas empresas del sector manufacturero.

Tabla 1. Argentina y Ecuador: Distribución por sector manufacturero de la muestra (Panel 2006-2010). En porcentajes

\begin{tabular}{ccc}
\hline Sector Industrial (en porcentaje) & Argentina & Ecuador \\
\hline Alimentos & 23,72 & 27,78 \\
\hline Textiles & 12,82 & 11,11 \\
\hline Confecciones & 15,38 & 9,26 \\
\hline Productos Químicos & 10,26 & 7,41 \\
\hline Plásticos y Cauchos & 1,28 & 7,41 \\
\hline Productos minerales no metálicos & 0,32 & 1,01 \\
\hline Metales Básicos & $\ldots, \ldots$ \\
\hline Fabricación de productos metálicos & 0,32 & 11,11 \\
\hline Maquinaria y equipos & 8,01 & $\ldots \ldots$ \\
\hline Electrónicos & 18,91 & $\ldots$. \\
\hline Otros & 0,45 & 24,91 \\
\hline Nicroempresas $(<10$ empleados) & 8,53 & 18,52 \\
\hline Pequeñas (>=10<49 empleados) & 18,01 & 51,85 \\
\hline Medianas $(>=49<200$ empleados) & 53,37 & 29,63 \\
\hline Total número de empresas & 28,62 & 54 \\
\hline
\end{tabular}

Fuente: Elaboración propia con base en Enterprise Survey del Banco Mundial (Panel 2006-2010) a empresas del sector manufacturero en el Ecuador y la Argentina.

\subsection{Variables y herramientas de análisis}

La finalidad del estudio es identificar cómo las empresas manufactureras ecuatorianas y argentinas realizan esfuerzos de innovación y generan resultados dentro de las empresas y su posterior comparabilidad de resultados. Se utilizó a la innovación en el producto y en el proceso como una variable endógena que es considerada en un modelo de regresión con dos ecuaciones. El problema de endogeneidad aparece cuando una variable independiente está correlacionada con el error, que es la parte no observada de la regresión. En este caso, puede esperarse que la innovación sea una variable endógena a la perfomance de la empresa, ya que puede estar relacionada con factores no observables que influyen también en la perfomance. El problema de endogeneidad de los regresores se pueden controlar con el uso de variables instrumentales ${ }^{3} \mathrm{y}$ de regresiones en dos etapas.

La investigación utiliza como metodología un análisis multivariado. Para las variables cuantitativas de los efectos de la innovación se utiliza el siguiente modelo de regresión de dos ecuaciones (Cameron y Triveridi, 2009, p. 467):

$$
\begin{array}{cc}
\text { Ec. } 1 & y_{\mathrm{i}}=y_{i} \beta_{1}+X_{1 i} \beta_{2}+u_{i} \\
\text { Ec. } 2 & y_{i}=X_{1 i} \Pi_{1}+X_{2 i} \Pi_{2}+v_{i}
\end{array}
$$

(1) La etiqueta del panel “ 2006 y 2010 “ indica empresas entrevistadas en 2006 y 2010 solamente.

(2) Para la Argentina las regiones de muestreo son: Buenos Aires, Rosario, Mendoza, Córdoba; para el Ecuador las provincias de muestreo son Pichincha, Guayas y Azuay.

(3) Las variables endógenas equivalen a las variables dependientes de un modelo de regresión de una sola ecuación, y las exógenas a las variables $X$, o regresoras en tal modelo, en tanto las variables $X$ no estén correlacionadas con el término de error de esa ecuación. La variable instrumental es una variable representante para la variable regresada rezagada pero con la propiedad que no está correlacionada con el error (Gujarati \& Porter, 2009). 
Donde

$y_{i}$ es la variable dependiente para la observación $i$-ésima (efecto de la innovación), $y_{i}$ representa al regresor endógeno (innovación en producto/proceso), $X_{1 i}$ representa los regresores exógenos incluidos, $X_{2 i}$ representa los regresores exógenos excluidos. $u_{i}$ y $v_{i}$ son los términos de error con media cero, y la correlación entre $u_{i}$ y los elementos de $v_{i}$, son presumibles diferentes a cero.

Para las variables cualitativas de los efectos de la innovación se controla la endogeneidad de la variable innovación en dos ecuaciones probit, bajo la forma de seemingly unrelated bivariate probit. La primera ecuación estima la variable efecto de la innovación y la segunda ecuación, la probabilidad la innovación y sus determinantes. El modelo puede escribirse así según Baum (2006, p. 271):

$$
\begin{array}{ll}
\text { Ec. } 1 & y_{1}^{*}=X_{1} \beta_{1}+u_{1} \\
\text { Ec. } 2 & y_{2}^{*}=X_{2} \beta_{2}+u_{2}
\end{array}
$$

Donde

$$
\left(\begin{array}{l}
u_{1} \\
u_{2}
\end{array}\right) \sim N\left\{\left(\begin{array}{l}
0 \\
0
\end{array}\right),\left(\begin{array}{ll}
1 & \rho \\
\rho & 1
\end{array}\right)\right\}
$$

Las variables observables a las dos variables latentes $y_{1}^{*}, y_{2}^{*}$ son $y_{1}$ , $y_{2}$.Estas variables $y_{1}, y_{2}$,toman el valor 1 si las correspondientes variables latentes $y_{1}^{*}, y_{2}^{*}$ toman valores positivos, y cero. Una formulación del modelo es el seemingly unrelated bivariate probit, donde dos ecuaciones probit son vistas como un sistema y deben estimarse en forma conjunta si $\rho \neq 0$. El no rechazo de la hipótesis nula conduce a la realización de una de un modelo probit simple.

En la Tabla 2 se presenta la descripción de las variables de innovación así como las variables utilizadas para instrumentar las mismas, en la primera etapa del modelo de dos ecuaciones, los determinantes de la innovación se identificaron en un estudio comparativo utilizando la Enterprise Survey del Banco Mundial del panel 2006-2010 (Astudillo y Briozzo, 2015) y se muestran en la ecuación 1.

Ec. 1 : Innovación Producto I010 $=f\left(I+D_{2006}, r_{r h h}{ }_{2006}\right.$, calidad $\left._{2006}, v_{i}\right)$

Ec. 1: Innovación Proceso ${ }_{2010}=f\left(I+D_{2006}, r r h h_{2006}\right.$, calidad $\left._{2006}, v_{i}\right)$

\begin{tabular}{|c|c|c|}
\hline Variables & Descripción & Tipo de Variables \\
\hline \multicolumn{3}{|c|}{ Variables Dependientes (2010) } \\
\hline $\begin{array}{l}\text { Innovación de } \\
\text { producto }\end{array}$ & $\begin{array}{l}\text { MIPYMES que introdujeron } \\
\text { un nuevo o significativamente } \\
\text { mejorado producto (bienes o } \\
\text { servicios), en los tres últimos } \\
\text { años }\end{array}$ & $\begin{array}{l}\text { Categórica } \\
1 \text { (Sí) } 0 \text { (No) }\end{array}$ \\
\hline $\begin{array}{l}\text { Innovación de } \\
\text { Proceso }\end{array}$ & $\begin{array}{l}\text { MIPYMES que realizaron un } \\
\text { nuevo o significativamente } \\
\text { mejorado proceso, en los tres } \\
\text { últimos años }\end{array}$ & $\begin{array}{l}\text { Categórica } \\
1 \text { (Sí) } 0 \text { (No) }\end{array}$ \\
\hline \multicolumn{3}{|c|}{ Variables Independientes (2006) } \\
\hline $\begin{array}{l}\text { Investigación y } \\
\text { desarrollo }\end{array}$ & $\begin{array}{l}\text { MIPYMES que realizaron } \\
\text { actividades de investigación y } \\
\text { desarrollo en el año fiscal }\end{array}$ & $\begin{array}{l}\text { Categórica } \\
1 \text { (Sí) } 0 \text { No }\end{array}$ \\
\hline Calidad & $\begin{array}{l}\text { MIPYMES que tienen una cer- } \\
\text { tificación de calidad reconocida } \\
\text { internacionalmente }\end{array}$ & $\begin{array}{l}\text { Categórica } \\
1 \text { (Sí) } 0 \text { No }\end{array}$ \\
\hline $\begin{array}{l}\text { Recursos hu- } \\
\text { manos califi- } \\
\text { cados }\end{array}$ & $\begin{array}{l}\text { MIPYMES que han realizado } \\
\text { programas de entrenamiento } \\
\text { para los trabajadores }\end{array}$ & $\begin{array}{l}\text { Categórica } \\
1 \text { (Sí) } 0(\mathrm{No})\end{array}$ \\
\hline
\end{tabular}

Tabla 2. Descripción de las variables dependientes, independientes y de control

Fuente: Astudillo y Briozzo, 2015

Posteriormente, la siguiente etapa de la ecuación consiste en estimar la siguiente ecuación, Ec. 2, empleando los resultados de la Ec. 1 para instrumentar la innovación:

Ec. 2: efecto $2010=\left(\right.$ estimaciones innovación producto y $\operatorname{proceso}($ Ec. 1$)$, variables control, $\left.\mu_{i}\right)$

En donde, los efectos de la innovación (performance empresarial) están en función de la innovación del producto y del proceso y necesitan de variables instrumentales que no estén correlacionadas con pero que estén correlacionadas con . Una variable que satisface estas condiciones es una variable instrumental que no esté correlacionada con el efecto de la innovación pero sí con la variable innovación.

Para estimar la Ec. 2, se utilizaron las siguientes variables en la segunda etapa de cada regresión: 
Variables Dependientes (2010) Efectos de la innovación en el producto y en el proceso

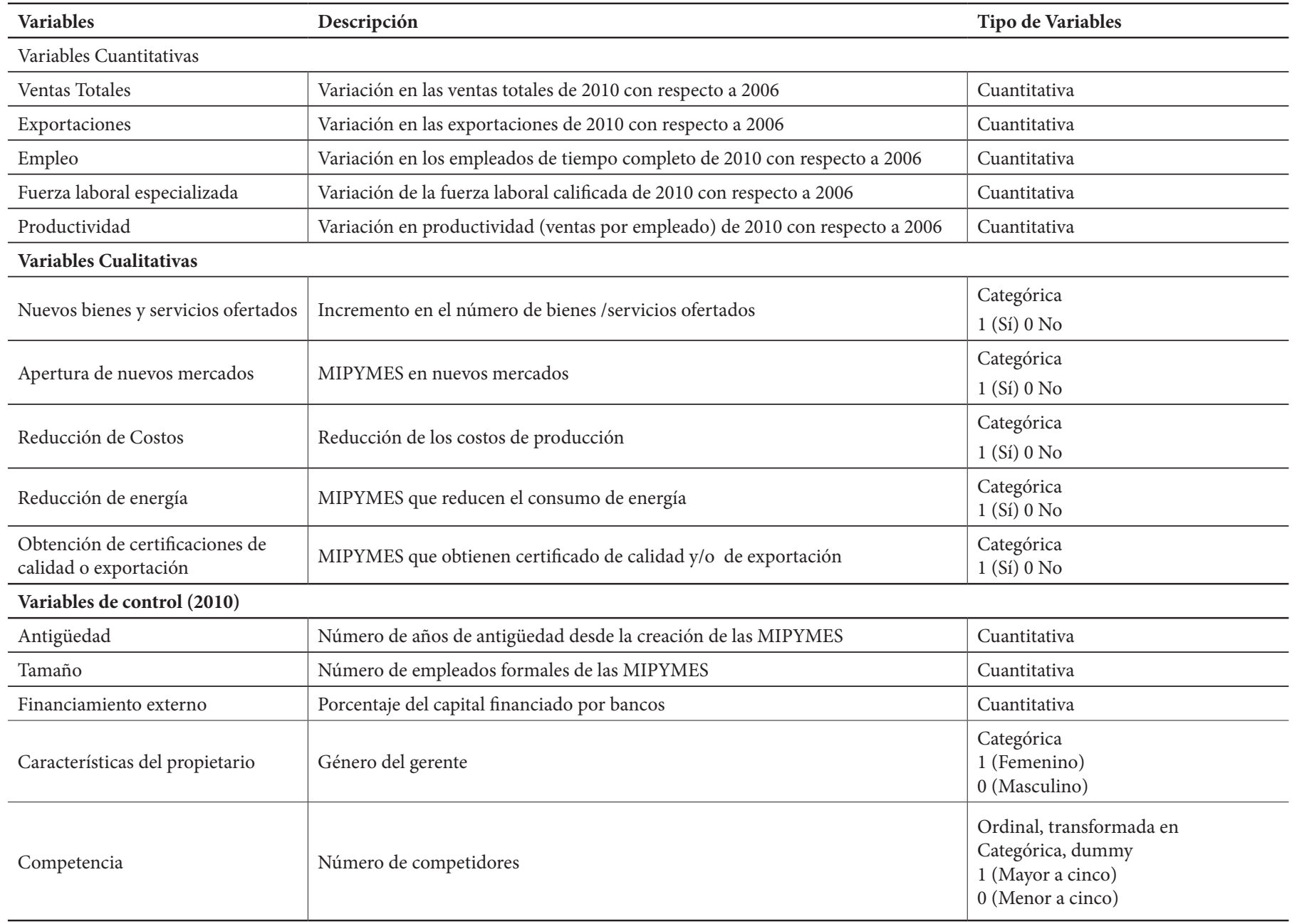

Fuente: Elaboración propia a partir de datos de la Enterprise Survey del Banco Mundial a empresas del sector manufacturero en la Argentina y en el Ecuador, Panel (2006-2010).

Para el análisis de la endogeneidad e la innovación en las variables cualitativas, se utiliza los tests de Durbin- Wu- Hausman (Baum, 2006 , p. 211), que consideran a la variable innovación como exógena. En este trabajo, para el análisis de endogeneidad se considera el test de Durbin que usa una estimación de la varianza del error basada en el modelo suponiendo que las variables que se están probando son exógenas. La aceptación de la hipótesis nula de exogeneidad de la innovación, es decir un test Durbin no significativo, conduce a la realización de una regresión en una sola etapa: la estimación de mediante mínimos cuadrados ordinarios. A su vez, la endogeneidad de la innovación para las variables cualitativas se estima con el test de la hipótesis nula de (Baum, 2006, p. 271). La aceptación de la hipótesis nula de exogeneidad, es decir un no significativo, conduce a la realización de una regresión en una etapa: la estimación mediante un modelo probit simple.

\section{Resultados}

Las Tablas 4 y 5 recogen las estimaciones de las variables desempeño cualitativas y cuantitativas de la innovación en el producto y en el proceso de las empresas manufactureras argentinas y ecuatorianas. Las sub-tablas A y B evidencian las variables desempeño cualitativas y presentan el p-value de la regresiones probit en dos etapas, la hipótesis nula rho $=0$ en la regresión biprobit y la regresión probit en una etapa en las últimas filas de las tablas. Se muestran los coeficientes de correlación y la significancia de las variables control del MCO de dos ecuaciones cuando rh $0=0$ es significante es decir se acepta la hipótesis de endogeneidad de la innovación. De lo contrario, se presentan los coeficientes de correlación y su significancia de una regresión probit en una etapa cuando tiene significancia estadística. 
Las variables desempeño cuantitativas se presentan en las sub-tablas C y D y presentan el p-value del MCO en dos etapas, el test Durbin de endogeneidad y el MCO en una etapa en las últimas filas de las tablas. Se muestran los coeficientes de correlación y la significancia de las variables control del MCO de dos ecuaciones cuando el test Durbin es significante es decir se acepta la hipótesis de endogeneidad de la innovación. De lo contrario, se presentan los coeficientes de correlación y su significancia de un MCO de una etapa con su significancia estadística.

El Anexo 1 recoge los estadísticos descriptivos de las variables dependientes, independientes y de control y el Anexo 2 las preguntas de los efectos de la innovación y su codificación de la Enterprise Survey del Banco Mundial.

\subsection{Efectos de la innovación en la Argentina}

Tabla 4. Estimaciones para la innovación en el producto y en el proceso para la Argentina- variables de la perfomance empresarial cualitativas y cuantitativas Tabla 4A. Argentina: Efectos cualitativos de la innovación en el producto

\begin{tabular}{|c|c|c|c|c|c|c|}
\hline Efectos/Variables control & $\begin{array}{l}\text { Nuevos } \\
\text { Mercados }\end{array}$ & $\begin{array}{l}\text { Incremento en el número } \\
\text { de bienes ofrecidos }\end{array}$ & $\begin{array}{l}\text { Mejora de la } \\
\text { calidad }\end{array}$ & $\begin{array}{l}\text { Reducción de } \\
\text { Costos }\end{array}$ & $\begin{array}{l}\text { Reducción } \\
\text { energía }\end{array}$ & $\begin{array}{l}\text { Obtención de certificaciones } \\
\text { de calidad o exportación }\end{array}$ \\
\hline Innovación en el producto & $\begin{array}{l}1,4592^{*} \\
(0,000)\end{array}$ & $\begin{array}{l}2.0368^{*} \\
(0,000)\end{array}$ & $\begin{array}{l}2,2845^{*} \\
(0,000)\end{array}$ & $\begin{array}{l}1,8499^{*} \\
(0,000)\end{array}$ & $\begin{array}{l}0,0229 \\
(0,930)\end{array}$ & $\begin{array}{l}1,5676^{*} \\
(0,000)\end{array}$ \\
\hline Antigüedad & $\begin{array}{l}0,0051 \\
(0,106)\end{array}$ & $\begin{array}{l}-0,0160^{\star *} \\
(0,067)\end{array}$ & $\begin{array}{l}0,0035^{\star *} \\
(0,083)\end{array}$ & $\begin{array}{l}0,0042 \\
(0,219)\end{array}$ & $\begin{array}{l}0,0020 \\
(0,638)\end{array}$ & $\begin{array}{l}-0,0012 \\
(0,743)\end{array}$ \\
\hline Tamaño & $\begin{array}{l}0,0022 \\
(0,143)\end{array}$ & $\begin{array}{l}0,0016 \\
(0,648)\end{array}$ & $\begin{array}{l}-0,0000 \\
(0,969)\end{array}$ & $\begin{array}{l}0,0016 \\
(0,462)\end{array}$ & $\begin{array}{l}0,0009 \\
(0,687)\end{array}$ & $\begin{array}{l}0,0027 \\
(0,165)\end{array}$ \\
\hline Financiamiento externo & $\begin{array}{l}0,0044 \\
(0,305)\end{array}$ & $\begin{array}{l}0,0129 \\
(0,507)\end{array}$ & $\begin{array}{l}0,0093 \\
(0,171)\end{array}$ & $\begin{array}{l}0,0005 \\
(0,908)\end{array}$ & $\begin{array}{l}-0,0016 \\
(0,814)\end{array}$ & $\begin{array}{l}0,0048 \\
(0,229)\end{array}$ \\
\hline Género del gerente & $\begin{array}{l}-0,0419 \\
(0,919)\end{array}$ & $\begin{array}{l}-0,1063 \\
(0,760)\end{array}$ & $\begin{array}{l}-0,1739 \\
(0,598)\end{array}$ & $\begin{array}{l}0,0770 \\
(0,812)\end{array}$ & $\begin{array}{l}-0,4716 \\
(0,374)\end{array}$ & $\begin{array}{l}-0,2911 \\
(0,158)\end{array}$ \\
\hline Competencia & $\begin{array}{l}-0,0372 \\
(0,291)\end{array}$ & $\begin{array}{l}0,0881 \\
(0,413)\end{array}$ & $\begin{array}{l}-0,0094 \\
(0,825)\end{array}$ & $\begin{array}{l}-0,0056 \\
(0,908)\end{array}$ & $\begin{array}{l}0,0336 \\
(0,550)\end{array}$ & $\begin{array}{l}-0,0450 \\
(0,161)\end{array}$ \\
\hline $\begin{array}{l}\text { Biprobit } \\
\text { Prob }>\text { F }\end{array}$ & $0,0000^{*}$ & $0,0025^{*}$ & $0,0000^{*}$ & $0,0000^{*}$ & 0,8050 & $0,0000^{*}$ \\
\hline $\begin{array}{l}\text { Likelihood-ratio test para } \\
\text { rho }=0\end{array}$ & $0,0922^{*}$ & $0,0940^{* *}$ & $0,0198^{*}$ & $0,0979^{*}$ & 0,6182 & $0,0001^{*}$ \\
\hline $\begin{array}{l}\text { Probit } \\
\text { Prob }>F\end{array}$ & $\cdots \cdots$ & $\cdots \cdots$ & $\cdots \cdots$ & $\cdots \cdots$ & 0,9352 & $\ldots \ldots$ \\
\hline
\end{tabular}

Fuente: Elaboración propia a partir de datos de la Enterprise Survey del Banco Mundial a empresas del sector manufacturero en la Argentina y en el Ecuador, Panel (2006-2010).

${ }^{*}$ significancia al $0,05,{ }^{* *}$ significancia al 0,10 (p-values entre paréntesis)

Para la Argentina, la Tabla 4A evidencia que los resultados de las regresiones probit (biprobit) en dos etapas son globalmente significativas para las variables de la performance empresarial cualitativas: Nuevos Mercados, Incremento de Bienes Ofrecidos, Mejora de la Calidad, Reducción de Costos y Obtención de Certificaciones de Calidad o Exportación. Se rechaza la hipótesis de exogeneidad de la innovación para estas variables dependientes, por lo que estas variables se analizaron con las regresiones probit en dos etapas. La variable Reducción de Energía no es significante en la estimación de dos re- gresiones y no se rechaza la hipótesis nula de exogeneidad de la innovación, por lo que se analiza con una regresión en una etapa, que resultó no significativa globalmente. Los resultados muestran un efecto positivo de la variable innovación en el producto de manera individual en Nuevos Mercados, Incremento en el Número de Benes Ofrecidos, Mejora de la Calidad y Reducción de Costos. La única variable control significativa es la antigüedad, con un efecto negativo sobre el Incremento en el Número de Benes Ofrecidos y positivo en la Mejora de Calidad. 
Tabla 4B. Argentina: Efectos cualitativos de la innovación en el proceso

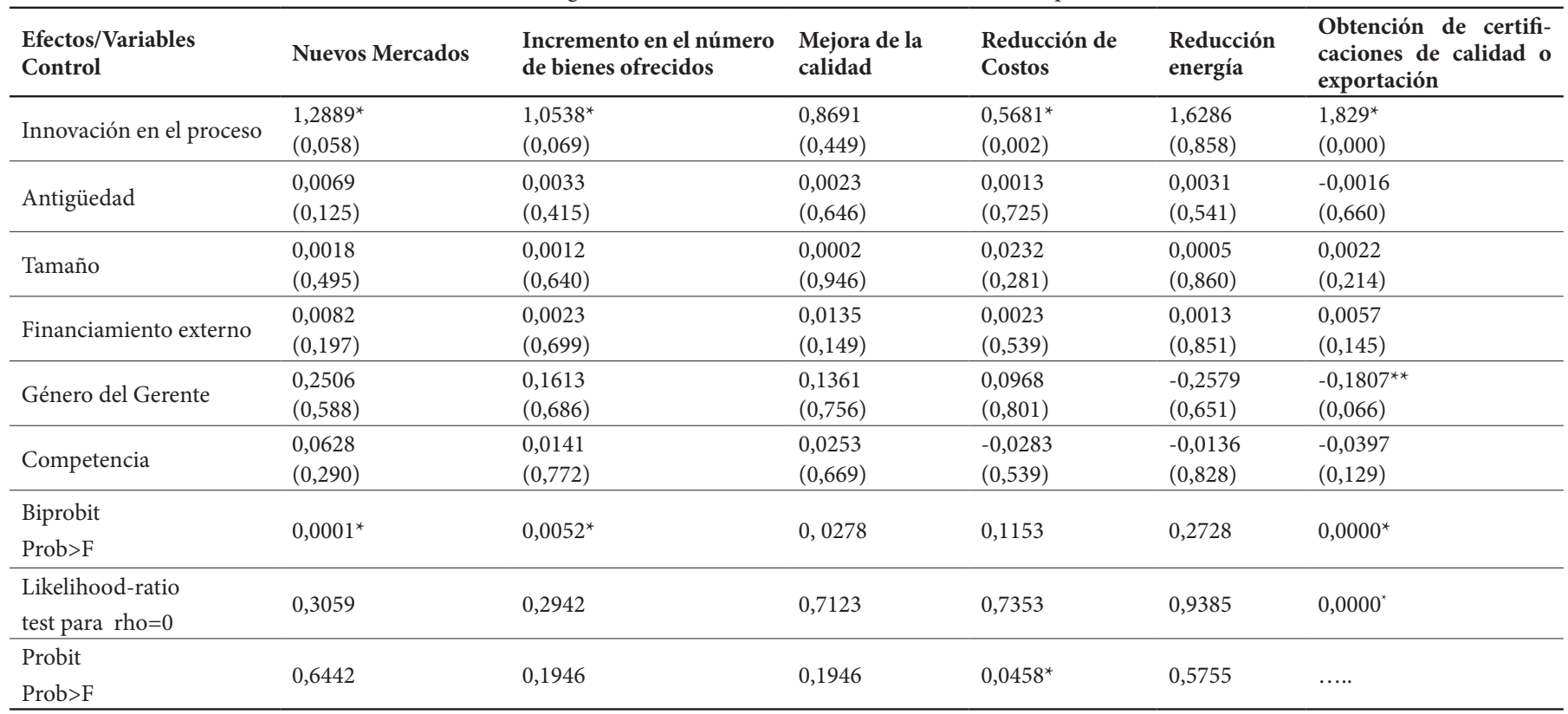

Fuente: Elaboración propia a partir de datos de la Enterprise Survey del Banco Mundial a empresas del sector manufacturero en la Argentina y en el Ecuador, Panel (2006-2010).

${ }^{*}$ significancia al $0,05,{ }^{* *}$ significancia al 0,10 (p-values entre paréntesis)

La Tabla 4B para la Argentina muestra que se rechaza la hipótesis nula de exogeneidad de la innovación únicamente para la variable Obtención de Certificaciones de Calidad o Exportación. En este caso, los resultados de la regresión en dos etapas muestran un efecto positivo de la innovación en proceso, junto con la incidencia negativa de contar con una gerente mujer. Este resultado puede explicarse por diferencias de los sectores productivos en los cuales predomina la contratación de hombres.
El resto de las variables resultados fueron estimadas mediante regresiones probit simples. Los resultados son globalmente significativos para la Reducción en costos, donde la innovación en proceso tiene efecto positivo. Para las restantes variables resultados (Nuevos Mercados, Incremento en el Número de Bienes Ofrecidos, Mejora de la Calidad y Reducción de Energía), los resultados son globalmente no significativos.

Tabla 4C. Argentina: Efectos cuantitativos de la innovación en el producto

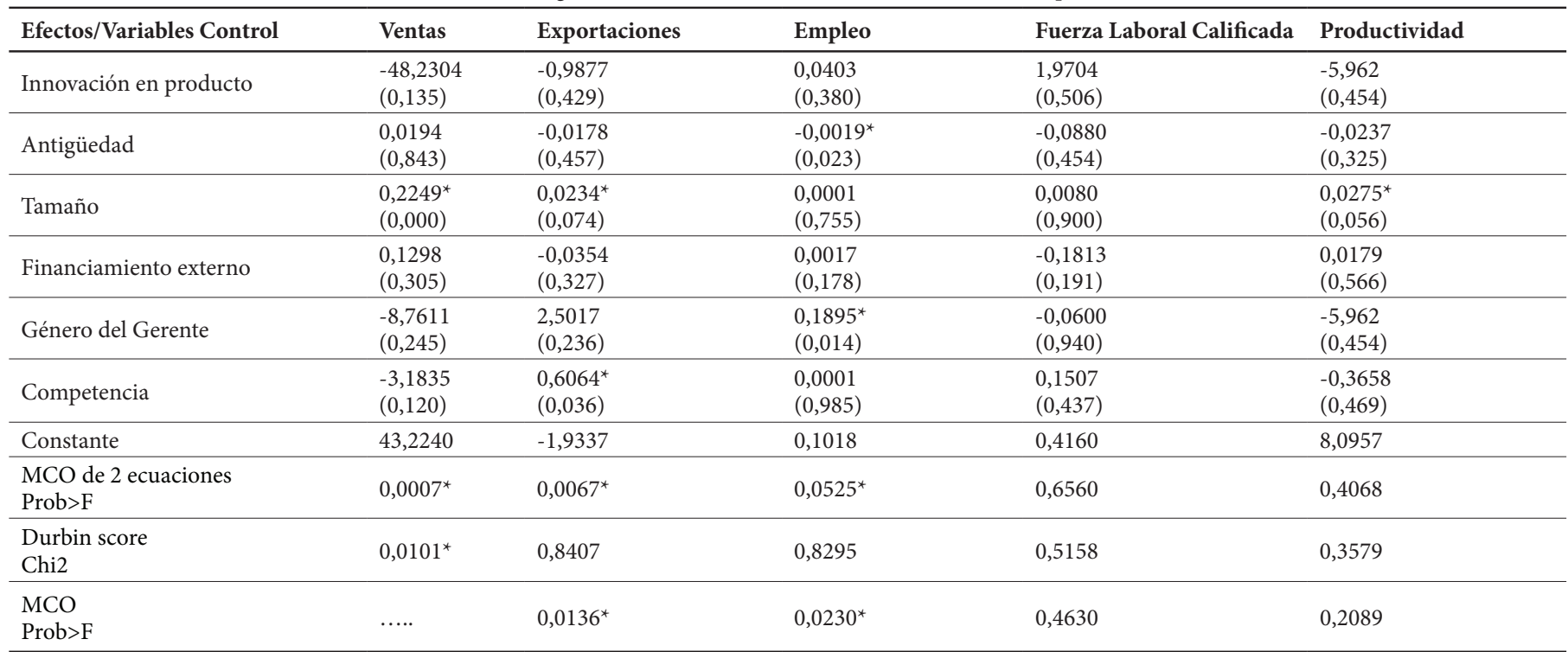

Fuente: Elaboración propia a partir de datos de la Enterprise Survey del Banco Mundial a empresas del sector manufacturero en la Argentina y en el Ecuador, Panel (2006-2010).

*significancia al 0,05, **significancia al 0,10 (p-values entre paréntesis) 
Para la Argentina, la Tabla 4C muestra regresiones globalmente significativas en 2 etapas (ivregress) para las Ventas, las Exportaciones y el Empleo. En cambio, para la Fuerza Laboral Calificada y la Productividad las regresiones en 2 etapas no son en conjunto significativas. Como se rechaza la hipótesis de exogeneidad de la innovación sólo para las Ventas, esta variable dependiente se analizó con la regresión en 2 etapas, y el resto de las variables de la performance empresarial se estudian con una regresión en una etapa. Los resultados de las regresiones en una etapa son conjuntamente significativos para Ex- portaciones y Empleo, y resultan no significativos para Fuerza Laboral Calificada y Productividad.

La innovación en producto no presenta efectos significativos para ninguna de las estimaciones. En cuanto a las variables control, el tamaño tiene una incidencia positiva en las Ventas y Exportaciones, mientras que la antigüedad incide negativamente en el Empleo. Contar con una gerente mujer incide positivamente en el Empleo, y una elevada competencia tiene un efecto positivo en las Exportaciones.

Tabla 4D. Argentina: Efectos cuantitativos de la innovación en el proceso

\begin{tabular}{|c|c|c|c|c|c|}
\hline Efectos/Variables control & Ventas & Exportaciones & Empleo & Fuerza Laboral Calificada & Productividad \\
\hline Innovación en proceso & $\begin{array}{l}0,7788 \\
(0,761)\end{array}$ & $\begin{array}{l}8,5474^{\star *} \\
(0,069)\end{array}$ & $\begin{array}{l}0,1010^{*} \\
(0,011)\end{array}$ & $\begin{array}{l}-0,9330 \\
(0,498)\end{array}$ & $\begin{array}{l}-0,7403 \\
(0,812)\end{array}$ \\
\hline Antigüedad & $\begin{array}{l}0,0331 \\
(0,564)\end{array}$ & $\begin{array}{l}0,0303 \\
(0,399)\end{array}$ & $\begin{array}{l}-0,0019 \\
(0,021)^{*}\end{array}$ & $\begin{array}{l}-0,1283 \\
(0,158)\end{array}$ & $\begin{array}{l}-0,0191 \\
(0,380)\end{array}$ \\
\hline Tamaño & $\begin{array}{l}0,2262^{*} \\
(0,000)\end{array}$ & $\begin{array}{l}-0,0461^{\star} \\
(0,012)\end{array}$ & $\begin{array}{l}0,0000 \\
(0,894)\end{array}$ & $\begin{array}{l}0,0020 \\
(0,702)\end{array}$ & $\begin{array}{l}0,0229 \\
(0,380)\end{array}$ \\
\hline Género del Gerente & $\begin{array}{l}-3,3768 \\
(0,500)\end{array}$ & $\begin{array}{l}4,1121 \\
(0,120)\end{array}$ & $\begin{array}{l}0,2019^{*} \\
(0,008)\end{array}$ & $\begin{array}{l}-0,0364 \\
(0,715)\end{array}$ & $\begin{array}{l}2,0772 \\
(0,236)\end{array}$ \\
\hline Competencia & $\begin{array}{l}0,7783 \\
(0,906)\end{array}$ & $\begin{array}{l}0,7339^{*} \\
(0,034)\end{array}$ & $\begin{array}{l}-0,0017 \\
(0,864)\end{array}$ & $\begin{array}{l}0,0354 \\
(0,715)\end{array}$ & $\begin{array}{l}-0,0403 \\
(0,862)\end{array}$ \\
\hline Durbin score (chi2) & 0,7132 & $0,0264^{*}$ & 0,3242 & 0,3110 & 0,8864 \\
\hline $\begin{array}{l}\mathrm{MCO} \\
\text { Prob }>\mathrm{F}\end{array}$ & $0,0000^{*}$ & $\ldots$ & $0,0023^{*}$ & 0,4552 & 0,2483 \\
\hline
\end{tabular}

Fuente: Elaboración propia a partir de datos de la Enterprise Survey del Banco Mundial a empresas del sector manufacturero en la Argentina y en el Ecuador, Panel (2006-2010).

*significancia al 0,05, ${ }^{* *}$ significancia al 0,10 (p-values entre paréntesis)

En la Tabla 4D, se observan regresiones globalmente significativas en 2 etapas (ivregress) para las Ventas, las Exportaciones y el Empleo. En cambio, para la Fuerza Laboral Calificada y la Productividad las regresiones en 2 etapas no son conjuntamente significativas. Como se rechaza la hipótesis nula de exogeneidad de la innovación para las Ventas y las Exportaciones, estas variables dependientes se analizaron con la regresión en 2 etapas, y el resto de las variables de la performance empresarial se analizan con una regresión en una etapa. Los resultados de las regresiones en una etapa son conjuntamente signifi- cativos para las variables Ventas y Empleo, y resultan no significativos para Fuerza Laboral Calificada y Productividad.

Los resultados muestran un efecto positivo de la innovación en proceso en las Exportaciones y en el Empleo. Con respecto a las variables control, sobre las Ventas tiene un efecto positivo el tamaño de la empresa. En las Exportaciones incide positivamente la competencia, y de manera negativa el tamaño, y en el Empleo incide positivamente el género del propietario y de manera negativa la antigüedad de la empresa. 


\subsection{Efectos de la innovación en el Ecuador}

Tabla 5. Estimaciones para la innovación en el producto y la innovación en el proceso para el Ecuador- variables de la perfomance empresarial cualitativas y cuantitativas

Tabla 5A. Ecuador: Efectos cualitativos de la innovación en el producto

\begin{tabular}{|c|c|c|c|c|c|c|}
\hline Efectos/Variable control & Nuevos Mercados & $\begin{array}{l}\text { Incremento en } \\
\text { el número de } \\
\text { bienes ofrecidos }\end{array}$ & $\begin{array}{l}\text { Mejora de la } \\
\text { calidad }\end{array}$ & $\begin{array}{l}\text { Reducción de } \\
\text { Costos }\end{array}$ & $\begin{array}{l}\text { Reducción } \\
\text { energía }\end{array}$ & $\begin{array}{l}\text { Obtención de certificacio- } \\
\text { nes de calidad o exporta- } \\
\text { ción }\end{array}$ \\
\hline Innovación en el producto & - & - & $\begin{array}{l}-0,5662 \\
(0,312)\end{array}$ & $\begin{array}{l}1,2630^{*} \\
(0,002)\end{array}$ & $\begin{array}{l}1,5714^{*} \\
(0,000)\end{array}$ & - \\
\hline Antigüedad & $\begin{array}{l}-0,0098 \\
(0,1688)\end{array}$ & $\begin{array}{l}0,0255 \\
(0,471)\end{array}$ & $\begin{array}{l}-0,0000 \\
(0,997)\end{array}$ & $\begin{array}{l}0,0020 \\
(0,865)\end{array}$ & $\begin{array}{l}0,0142^{*} \\
(0,058)\end{array}$ & $\begin{array}{l}0,0057 \\
(0,726)\end{array}$ \\
\hline Tamaño & $\begin{array}{l}0,0006 \\
(0,940) \\
\end{array}$ & $\begin{array}{l}0,0958 \\
(0,426)\end{array}$ & $\begin{array}{l}-0,0055 \\
(0,352)\end{array}$ & $\begin{array}{l}-0,0045 \\
(0,387)\end{array}$ & $\begin{array}{l}0,0040 \\
(0,221)\end{array}$ & $\begin{array}{l}-0,0016 \\
(0,862)\end{array}$ \\
\hline Financiamiento externo & $\begin{array}{c}-0,0286 \\
(0,138)\end{array}$ & $\begin{array}{l}-0,0145 \\
(0,205)\end{array}$ & $\begin{array}{c}-0,0099 \\
(0,939)\end{array}$ & $\begin{array}{c}-0,0048 \\
(0,730)\end{array}$ & $\begin{array}{l}0,0102 \\
(0,404)\end{array}$ & $\begin{array}{l}0,0004 \\
(0,970)\end{array}$ \\
\hline Género del Gerente & $\begin{array}{l}-0,4287 \\
(0,595)\end{array}$ & $\begin{array}{l}0,1116 \\
(0,889)\end{array}$ & $\begin{array}{l}0,6178 \\
(0,315)\end{array}$ & $\begin{array}{l}0,6147 \\
(0,238)\end{array}$ & $\begin{array}{l}0,5457 \\
(0,289)\end{array}$ & $\begin{array}{l}0,1550 \\
(0,852)\end{array}$ \\
\hline Competencia & $\begin{array}{l}-0,1017 \\
(0,546)\end{array}$ & $\begin{array}{c}-0,1135 \\
(0,511)\end{array}$ & $\begin{array}{c}-0,0099 \\
(0,939)\end{array}$ & $\begin{array}{l}-0,0590 \\
(0,675)\end{array}$ & $\begin{array}{l}0,1195 \\
(0,247)\end{array}$ & $\begin{array}{c}-0,1953 \\
(0,239)\end{array}$ \\
\hline $\begin{array}{l}\text { Biprobit } \\
\text { Prob }>\text { F }\end{array}$ & 0,8822 & 0,5944 & $0,0002^{*}$ & $0,0112^{*}$ & $0,0728^{*}$ & $\cdots \cdots$ \\
\hline $\begin{array}{l}\text { Likelihood-ratio test para } \\
\text { rho }=0\end{array}$ & 0,9850 & 0,1816 & 0,1835 & $0,0244^{*}$ & $0,0313^{*}$ & $\ldots \ldots$ \\
\hline $\begin{array}{l}\text { Probit } \\
\text { Prob }>\text { F }\end{array}$ & 0,6805 & 0,9239 & 0,2988 & $\cdots \cdots$ & $\cdots \cdots$ & 0,8115 \\
\hline
\end{tabular}

Fuente: Elaboración propia a partir de datos de la Enterprise Survey del Banco Mundial a empresas del sector manufacturero en la Argentina y en el Ecuador, Panel (20062010). ${ }^{*}$ significancia al $0,05,{ }^{* *}$ significancia al 0,10 (p-values entre paréntesis). Nota: En la estimación de las variables resultado Nuevos Mercados, Incremento de Bienes Ofrecidos y Obtención de certificaciones de calidad o exportación, la variable Innovación en el Producto se omitió por su variabilidad y por el tamaño de la muestra.

Para el Ecuador, la Tabla 5A muestra regresiones globalmente significativas en 2 etapas (biprobit) para la Reducción de Energía (consumo de gas, electricidad, etc.) y para la Reducción de Costos. Dado que se rechaza la hipótesis nula de exogeneidad de la innovación, estas variables se analizaron con la regresión en dos etapas. Los resultados muestran que la innovación en el producto está relacionada positivamente con la Reducción de Energía y la Reducción de Costos. En las regresiones restantes (Mejora de la Calidad), la innovación en el producto no resulta significativa. Con respecto a las variables control, se observa que la antigüedad tiene un efecto positivo en la Reducción de Energía.

Tabla 5B. Ecuador: Efectos cualitativos de la innovación en el proceso

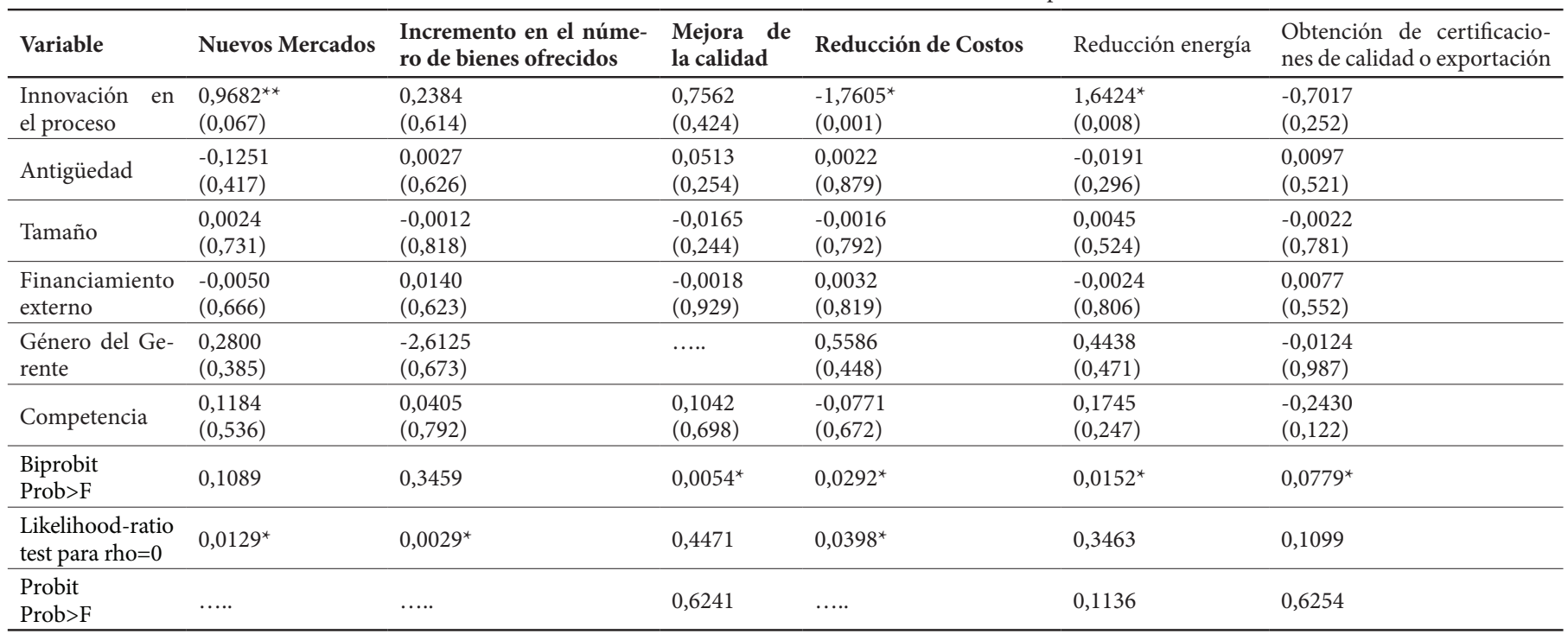

Fuente: Elaboración propia a partir de datos de la Enterprise Survey del Banco Mundial a empresas del sector manufacturero en la Argentina y en el Ecuador, Panel (2006-2010). * significancia al 0,05, ${ }^{* *}$ significancia al 0,10 (p-values entre paréntesis). Nota: En la estimación de las variables resultado Mejora de la Calidad se omitió la variable género del gerente por su variabilidad y por el tamaño de la muestra. 
En relación a los efectos cualitativos de la innovación en el proceso para el Ecuador, la Tabla 5B muestra que se rechaza la hipótesis de exogeneidad para las variables Nuevos Mercados, Incremento de Bienes Ofrecidos y Reducción de Costos, siendo esta última la única regresión globalmente significativa en dos etapas. En este caso, se observa un efecto negativo de la innovación en proceso sobre la Reducción de Costos. Las restantes variables resultado (Mejora de la Calidad, Reducción de Energía y la Obtención de Certificaciones de Calidad o Exportación) se estiman en una etapa y no resultaron globalmente significativas.

Tabla 5C. Ecuador Efectos cuantitativos de la innovación en el producto

\begin{tabular}{|c|c|c|c|c|c|}
\hline Efectos/Variables control & Ventas & Exportaciones & Empleo & Fuerza Laboral Calificada & Productividad \\
\hline Innovación en el producto & $\begin{array}{l}-59,7717 \\
(0,158)\end{array}$ & $\begin{array}{l}-13,4531 \\
(0,693)\end{array}$ & $\begin{array}{l}0,4600 \\
(0,522)\end{array}$ & $\begin{array}{l}2,0198 \\
(0,423)\end{array}$ & $\begin{array}{l}-1,4287 \\
(0,535)\end{array}$ \\
\hline Antigüedad & $\begin{array}{l}0,1112 \\
(0,750)\end{array}$ & $\begin{array}{l}0,0534 \\
(0,820)\end{array}$ & $\begin{array}{l}-0,0012 \\
(0,819)\end{array}$ & $\begin{array}{l}-0,0078 \\
(0,714)\end{array}$ & $\begin{array}{l}-0,0029 \\
(0,878)\end{array}$ \\
\hline Tamaño & $\begin{array}{l}0,2687^{*} \\
(0,048)\end{array}$ & $\begin{array}{l}-0,0360 \\
(0,688)\end{array}$ & $\begin{array}{l}-0,0002 \\
(0,839)\end{array}$ & $\begin{array}{l}-0,0063 \\
(0,522)\end{array}$ & $\begin{array}{l}0,0146^{*} \\
(0,048)\end{array}$ \\
\hline Financiamiento externo & $\begin{array}{l}0,2869 \\
(0,479)\end{array}$ & $\begin{array}{l}0,1460 \\
(0,632)\end{array}$ & $\begin{array}{l}-0,0055 \\
(0,393) \\
\end{array}$ & $\begin{array}{l}0,0008 \\
(0,975)\end{array}$ & $\begin{array}{l}-0,0035 \\
(0,873) \\
\end{array}$ \\
\hline Competencia & $\begin{array}{l}1,4728 \\
(0,604)\end{array}$ & $\begin{array}{l}-2010 \\
(0,854)\end{array}$ & $\begin{array}{l}-0,0339 \\
(0,819)\end{array}$ & $\begin{array}{l}-0,0606 \\
(0,766)\end{array}$ & $\begin{array}{l}-0,0508 \\
(0,742)\end{array}$ \\
\hline Constante & 22,6016 & 6,3627 & $-0,0579$ & 1,2237 & 1,8937 \\
\hline $\begin{array}{l}\text { MCO de } 2 \text { ecuaciones } \\
\text { Prob }>\text { F }\end{array}$ & $0,0124^{*}$ & 0,9753 & 0,8310 & 0,5929 & 0,1371 \\
\hline $\begin{array}{l}\text { Durbin score } \\
\text { Chi2 }\end{array}$ & $0,0763^{\star *}$ & 0,7200 & 0,3317 & 0,6097 & 0,3680 \\
\hline
\end{tabular}

Fuente: Elaboración propia a partir de datos de la Enterprise Survey del Banco Mundial a empresas del sector manufacturero en la Argentina y en el Ecuador, Panel (2006-2010). * significancia al 0,05, ${ }^{* *}$ significancia al 0,10 (p-values entre paréntesis)

En la Tabla 5C, para el Ecuador la innovación en el producto muestra regresiones de 2 etapas (ivregress) con una significancia global únicamente en las Ventas, donde se rechaza además la exogeneidad de la variable innovación. Sin embargo, la innovación no resulta individualmente significativa. La variable control con relevancia es el tamaño de la empresa, con una relación positiva con las Ventas.
Las variables Exportaciones, Empleo, Fuerza Laboral Calificada, y Productividad no resultaron significantes en las regresiones de 2 etapas y no se rechaza la hipótesis de exogeneidad de la innovación, por lo que estas variables se estudiaron en una regresión en una etapa. Las regresiones así estimadas no resultaron conjuntamente significativas.

Tabla 5D. Ecuador Efectos cuantitativos de la innovación en el proceso

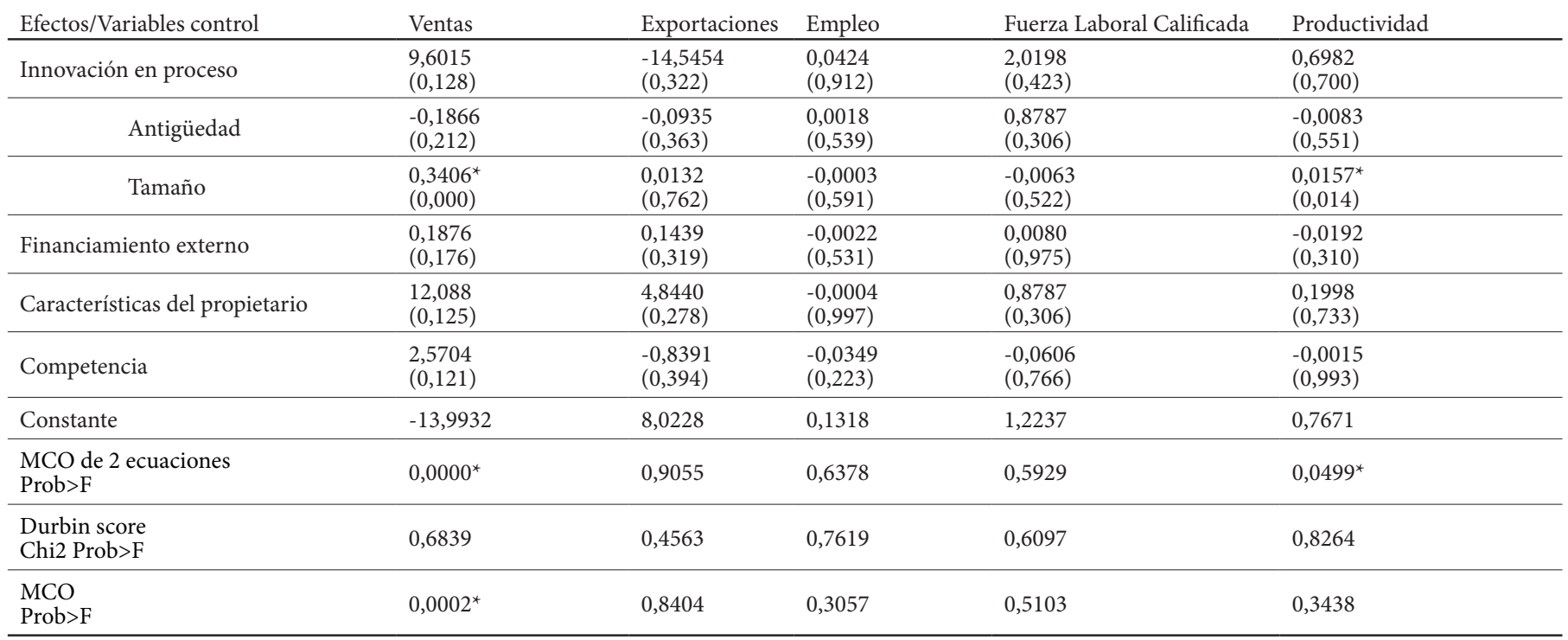

Fuente: Elaboración propia a partir de datos de la Enterprise Survey del Banco Mundial a empresas del sector manufacturero en la Argentina y en el Ecuador, Panel (2006-2010). * significancia al 0,05, ${ }^{*}$ significancia al 0,10 (p-values entre paréntesis) 
Los resultados de la Tabla 5D indican que en todos los casos analizados, no se rechaza la hipótesis de exogeneidad de la innovación, por lo cual se presentan las estimaciones en una etapa. La única regresión globalmente significativa tiene como variable dependiente a las Ventas, encontrándose que la innovación en el proceso no resulta significativa, mientras que el tamaño tiene un efecto positivo.
En la Tabla 6 se resumen los resultados de las estimaciones que muestran un efecto positivo de la innovación tanto en el producto como en el proceso sobre las variables desempeño, así como un efecto negativo de la innovación en el proceso.

Tabla 6. Argentina y Ecuador: Comparación de efectos de la innovación del producto y proceso

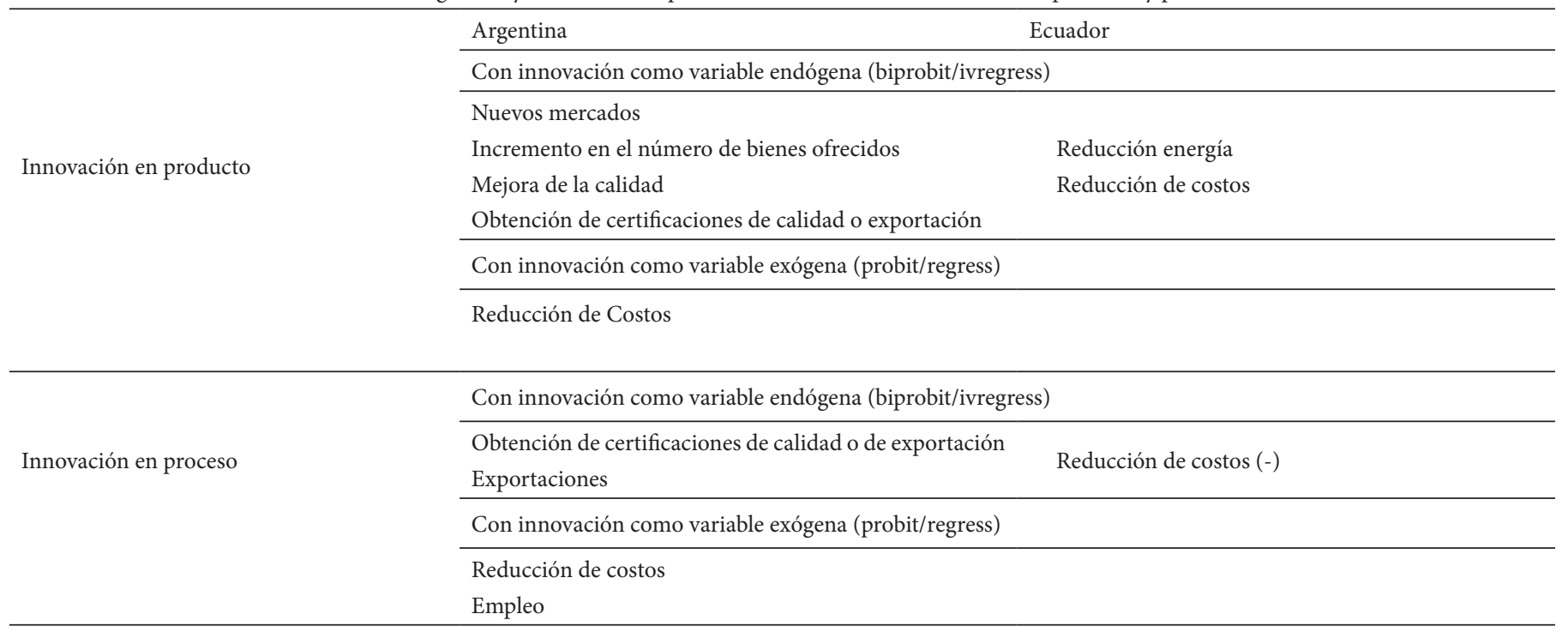

Fuente: Tablas 4 y 5

\section{Discusión}

Los resultados del estudio evidencian que la innovación en el proceso genera un impacto positivo en el Empleo con un modelo probit simple en la nación Argentina, contrario a lo planteado por Pianta (2003) que señala un impacto negativo de la innovación del proceso en esta variable desempeño en un estudio en las naciones de Europa. De igual manera, en la Argentina existe un impacto de la innovación en el proceso en las Exportaciones, lo que corrobora el criterio de que los más innovadores son más propensos a exportar, evidenciadas por Caldera, 2010; Martins, Goméz-Araujo, \& Vaillant, 2015; Love \& Roper, 2015, autores que encuentran una relación positiva entre las exportaciones y la innovación.

El impacto de la apertura de Nuevos Mercados y el Incremento de Bienes Ofrecidos en la nación argentina coincide con lo planteado en la literatura empírica revisada en Lööf et al., 2001; Klomp y Van Leeuwen, 2001; Gómez \& Calvo, 2011; Gunday et al., 2011; Brown \& Guzmán, 2014, en investigaciones en otras naciones y de manera específica en la Argentina coincide con estudios realizados en esta nación por Lugones, Suárez, \& Gregorini, 2007; Lugones, Suárez, \& Le Clech, 2007; Anlló \& Suárez 2009.

El impacto Mejora de la Calidad y Obtención de Certificaciones de Calidad o Exportación en la Argentina y en el Ecuador coincide con lo manifestado por Prajogo \& Sohal, 2003; Thai Hoang, Igel, \& Laosirihongthong, 2006 en estudios empíricos en otras naciones.
La Reducción de Energía considerado por Gómez \& Calvo, 2011 como un objetivo del empresariado se revela en esta investigación como un efecto de la innovación en el producto en el Ecuador. En referencia a la reducción de costos, contrario a Božić \& Radas, 2006; Caldera, 2010, el empresariado manufacturero del Ecuador percibe que la innovación en el proceso no influye en la reducción de costos de manera positiva, sin embargo los datos mostrados en la Argentina coinciden con estos autores en la evidencia que la innovación en el producto tiene un efecto la reducción de costos.

\section{Conclusiones}

El análisis desarrollado en esta investigación evidencia que la innovación en el producto y la innovación en el proceso generan efectos en la performance empresarial de las empresas manufactureras argentinas y ecuatorianas. Se realizó el control de la endogeneidad de la innovación en tres años de gestión empresarial (2006-2010) con la inclusión de variables control como el tamaño, la antigüedad, el financiamiento externo, el género del gerente, la competencia, la investigación y desarrollo, la calidad y los recursos humanos calificados y las estimaciones muestran evidencia de impactos diferentes y similares en ambos países como resultado de su actividad innovadora en el producto o el proceso.

En lo que respecta a impactos diferentes con el control endógeno de la innovación, la innovación en el producto se evidencia en el Ecuador con una significancia positiva en la reducción del consumo de energía (gas, electricidad, etc.) y en la Argentina un efecto positivo en la 
apertura de nuevos mercados, la mejora de la calidad, el incremento en el número de bienes ofrecidos y la obtención de certificaciones de calidad o de exportación. El efecto semejante positivo de la innovación del producto que se observa en ambos países es la reducción de costos con un control endógeno de la innovación solamente en el Ecuador. Con referencia a la innovación en el proceso tras controlar su endogeneidad, el Ecuador muestra un efecto positivo en la reducción de energía (gas, electricidad, etc.) y en la Argentina, la innovación en el proceso genera un efecto positivo en la obtención de certificaciones de calidad o exportación y en las exportaciones. En el empleo la innovación en el proceso genera un efecto positivo en el empleo sin controlar su endogeneidad. Con respecto a los impactos similares, en ambos países se observa a la reducción de costos como un efecto, sin embargo en el Ecuador la significancia de la innovación en el proceso es negativa en este impacto y en la Argentina esta variable desempeño es significativa con un modelo probit simple. Las actividades de innovación realizadas por las mipymes manufactureras no presentaron efectos significativos sobre las ventas, la productividad y la fuerza laboral calificada en ambos países. Las variables control que tuvieron relevancia en el estudio fueron el tamaño de la empresa, la antigüedad, el género del gerente y el grado de competencia.

Es necesario valorar los esfuerzos por innovar en las empresas manufactureras en ambos países, sin embargo, en comparación con el Ecuador, la Argentina presenta mayores efectos en la performance empresarial, esta evidencia revela que las empresas manufactureras argentinas poseen mayores resultados de su actividad innovadora posiblemente por un esfuerzo mayor en la inversión en ciencia, tecnología e innovación. De esta manera, se sugiere que la nación ecuatoriana promueva y facilite los procesos de ciencia, tecnología e innovación mediante el apoyo al financiamiento, una política fiscal con incentivos, la inversión en $\mathrm{I}+\mathrm{D}+\mathrm{i}$, entre otros factores para la consecución de mayores impactos de la innovación empresarial.

Una de las limitaciones del estudio es que la Enterprise Survey del Banco Mundial ofrece un listado de variables desempeño de la innovación que satisface el estudio y la aplicación de los tests estadísticos, sin embargo los objetivos de los empresarios del sector manufacturero pueden ser más extensos al momento de evaluar los impactos de la innovación. Así también, por el número reducido de observaciones en el Ecuador se omitieron variables control por el grado de convergencia de los datos. Además, es importante aclarar que el tamaño de la muestra en Ecuador pudo ser una limitación para evidenciar un mayor número de efectos de la innovación en este país, sin embargo la información del Banco Mundial permitió generar un aporte a la comparabilidad de Ecuador con otro país de la región como la Argentina.

De hecho, resultaría interesante luego de analizar los inputs, los outputs y sus efectos en la performance empresarial de las empresas manufactureras entre el Ecuador y la Argentina, identificar los obstáculos que impiden el proceso innovador en estas naciones con diferente perfil de CTI, estudio que puede contribuir a la formulación de políticas públicas para la cooperación en el desarrollo de la innovación en ambos países y al planteamiento de líneas de actuación en torno a la mejora de la competitividad empresarial al interior de cada país.

\section{Bibliografía}

Aghion, P., Howitt, P., Brant-Collett, M., \& García-Peñalosa, C. (1998). Endogenous growth theory. Cambridge MA: The MIT Press.

Astudillo Durán, S., \& Briozzo, A. E. (2015). Factores determinantes de la innovación en las MIPYMES manufactureras de la Argentina y el Ecuador. FAEDPYME INTERNATIONAL REVIEW, 4(7), 53-65.

Anlló, G. \& Suárez, D. (2009). Innovación: Algo más que I+D. Evidencias Iberoamericanas a partir de las encuestas de innovación: Construyendo las estrategias empresarias competitivas. CEPAL-REDES. Recuperado el 15 de Septiembre de 2016 de http://www.oei.es/salactsi/ innova.pdf

Božić, L., \& Radas, S. (2006). The effects of innovation activities in SMEs in the Republic of Croatia. Croatian Economic Survey, (8), 33-52.

Brown, F., \& Guzmán, A. (2014). Innovation and Productivity across Mexican Manufacturing Firms. Journal of Technology Management \& Innovation, 9(4), 36-52. _https://doi.org/10.4067/s071827242014000400003

Cameron, A. C., and P.K. Triverdi (2005). Microeconomics: Methods and Applications. New York: Cambridge University Press

Baum, C. (2006). An Introduction to Modern Econometrics Using Stata. Stata Press, College Station, Texas.

Caldera, A. (2010). Innovation and exporting: evidence from Spanish manufacturing firms. Review of World Economics, 146(4), 657689. https://doi.org/10.1007/s10290-010-0065-7

Chudnovsky, D., Lopez, A., \& Pupato, G. (2004). Innovation and productivity: A study of Argentine manufacturing firms' behavior (1992-2001). Research Policy, 35(2), 266-288. https://doi.org/10.1016/j.respol.2005.10.002

Crépon, B., Duguet, E., \& Mairesse, J. (1998). Research, Innovation And Productivity: An Econometric Analysis at the Firm Level. Economics of Innovation and New Technology, 7(2), 115-158.

Crespi, G., \& Dutrénit, G. (2013). Políticas de ciencia, tecnología e innovación para el desarrollo. La experiencia latinoamericana (eds). México: Foro Consultivo Científico y Tecnológico, A.C.

Dosi, G., Freeman, C., Nelson, R., Silverberg, G., \& Soete, L. (1988). Technical Change and Economic Theory. London: Pinter.

Drucker, P. F. (1986). Innovation and Entrepreneurship: Practice and Principles. New York: Harper \& Row.

Durbin, J. (1954). Errors in Variables. Revue De L'Institut International De Statistique / Review of the International Statistical Institute, 22(1/3), 23-32. https://doi.org/10.2307/1401917 
Dutrénit, G., \& Katz, J. (2005). Introduction: Innovation, growth and development in Latin-America: Stylized facts and a policy agenda. Innovation, 7(2-3), 105-130.

Evangelista, R., \& Vezzani, A. (2010). The economic impact of technological and organizational innovations. A firm-level analysis. Research Policy, 39(10), 1253-1263.

Fagerberg, J., Mowery, David C., \& Nelson, R. (2004). The Oxford Handbook of Innovation. New York: Oxford University Press.

García Pérez De Lema, D., \& Gálvez Albarracín, E. J. (2012). Impacto de la innovación sobre el rendimiento de la MIPYME: un estudio empírico en Colombia. Estudios Gerenciales, 28(122). https://doi.org/10.1016/s0123-5923(12)70191-2

Gómez-Vieites, A., J.L. Calvo G. (2011). Innovación: Factor clave del éxito empresarial. Bogotá, Colombia: Ecoe Ediciones.

Griffith, R., Huergo, E., Mairesse, J., \& Peters, B. (2006). Innovation and productivity across four European countries. Oxford Review of Economic Policy, 22(4), 483-498. https://doi.org/10.3386/w12722

Grossman, G. M., \& Helpman, E. (1993). Innovation and growth in the global economy. Cambridge MA: The MIT Press.

Gujarati, D., \& Porter, D. (2009). Econometría. México: McGraw-Hill.

Gunday, G., Ulusoy, G., Kilic, K., \& Alpkan, L. (2011). Effects of innovation types on firm performance. International Journal of Production Economics, 133(2), 662-676. https://doi.org/10.1016/j.ijpe.2011.05.014

Hassan, M. U., Shaukat, S., Nawaz, M. S., \& Naz, S. (2013). Effects of innovation types on firm performance: An empirical study on Pakistan's manufacturing sector. Pakistan Journal of Commerce and Social Sciences, 7(2), 243-262.

Kline, S. J., \& Rosenberg, N. (1986). An overview of innovation. The Positive Sum Strategy: Harnessing technology for Economic Growth, Washington, D.C.: National Academy Press

Klomp, L., \& Van Leeuwen, G. (2001). Linking innovation and firm performance: a new approach. International Journal of the Economics of Business, 8(3), 343-364. https://doi.org/10.1080/13571510110079612

Lööf, H., Heshmati, A., Asplund, R., \& Nåås, S.-O. (2001). Innovation and performance in manufacturing industries: A comparison of the Nordic Countries. SSE/EFI Working Paper Series in Economics and Finance. Recuperado el 15 de Septiembre de 2016 de http://core.kmi. open.ac.uk/download/pdf/7097896.pdf

Lööf, H., \& Heshmati, A. (2002). Knowledge capital and performance heterogeneity: A firm-level innovation study. International Journal of Production Economics, 76(1), 61-85. http://dx.doi.org/10.1016/ S0925-5273(01)00147-5
Love, J. H., \& Roper, S. (2015). SME innovation, exporting and growth: A review of existing evidence. International Small Business Journal, 33(1), 28-48. https://doi.org/10.1177/0266242614550190

Lugones, G., Suárez, D., \& Gregorini, S. (2007). La innovación como fórmula para mejoras competitivas compatibles con incrementos salariales. Evidencias en el caso argentino. Centro Redes. Documento de trabajo, (36). Recuperado el 20 de Septiembre de 2016 de http://www.centroredes.org.ar/files/documentos/old/Doc.Nro36.pdf

Lugones, G., Suárez, D., \& Le Clech, N. (2007). Conducta innovativa y desempeño empresarial. Centro Redes. Documento (33). Recuperado el 20 de Septiembre de 2016 de http://www.centroredes.org.ar/files/ documentos/Doc.Nro33.pdf

Lundvall, B. (Ed.) (1992). National Systems of Innovation: Towards a Theory of Innovation and Interactive Learning. London: Pinter

Lundvall, B. (2009). Sistemas nacionales de innovación. Hacia una teoría de la innovación y el aprendizaje por interacción. Buenos Aires, Argentina: UNSAM.

Malerba, F., \& Orsenigo, L. (1996). Schumpeterian patterns of innovation are technology-specific. Research Policy, 25(3), 451-478. https://doi.org/ 10.1016/0048-7333(95)00840-3

Martins, I., Goméz-Araujo, E., \& Vaillant, Y. (2015). Mutual Effects Between Innovation Commitment and Exports: Evidence from the Owner-Manager in Colombia. Journal of Technology Management \& Innovation, 10(1), 103-116. http://dx.doi.org/10.4067/S071827242015000100008

Metcalfe, J. S. (1998). Evolutionary Economics and Creative Destruction. London, England: Psychology Press.

Nelson, R. R., \& Winter, S. G. (1982). An Evolutionary Theory of Economic Change. London, England: Harvard University Press.

OCDE. (2005). Manual de Oslo: Guía para la recogida e interpretación de datos sobre innovación. 3a ed. París, Francia: Organización de Cooperación y Desarrollo Económicos, Oficina de Estadísticas de las Comunidades Europeas.

Peters, B. (2008). Innovation and firm performance: An empirical investigation for German firms (Vol. 38). New York: Physica-Verlag, Heidelberg. http://dx.doi.org/ 10.1007/978-3-7908-2026-3

Pianta, M. (2003) Innovation and employment, in J. Fagerberg, D. Mowery and R. Nelson (eds), Handbook of Innovation, The Oxford University Press (forth), 1-33 pp.

Prajogo, D. I., \& Sohal, A. S. (2003). The relationship between TQM practices, quality performance, and innovation performance: An empirical examination. International Journal of Quality \& Reliability Management, 20(8), 901-918. http://dx.doi.org/10.1108/02656710310493625 
Reichert, F. M., \& Zawislak, P. A. (2014). Technological capability and firm performance. Journal of Technology Management \& Innovation, 9(4), 20-35. https://doi.org/10.4067/s0718-27242014000400002

Sandven, T., \& Smith, K. H. (2000). Innovation and economic performance at the enterprise level. France, 23- 24/11/2000.Conference innovation and enterprise creation: Statistics and indicators, Norway. Recuperado el 20 de Septiembre de 2016 de http://www.nifu.no/files/2012/11/STEPrapport10-2000.pdf

SENESCYT/INEC-Secretaría de Educación Superior, Ciencia, Tecnología e Innovación Ecuador-Instituto Nacional de Estadísticas y Censos (2014). Principales Indicadores de Actividades de Ciencia, Tecnología e Innovación, 93p.
Schumpeter, J. A. (1934). The theory of economic development. Cambridge: Harvard University Press, 255p.

Thai Hoang, D., Igel, B., \& Laosirihongthong, T. (2006). The impact of total quality management on innovation: findings from a developing country. International Journal of Quality \& Reliability Management, 23(9), 1092-1117._https://doi.org/10.1108/02656710610704230

Yeh-Yun Lin, C., \& Yi-Ching Chen, M. (2007). Does innovation lead to performance? An empirical study of SMEs in Taiwan. Management Research News, 30(2), 115-132. https://doi.org/10.1108/01409170710722955 


\section{Anexo No. 1}

Argentina y Ecuador: Estadísticos Descriptivos de los inputs, los outputs de la innovación y la performance empresarial

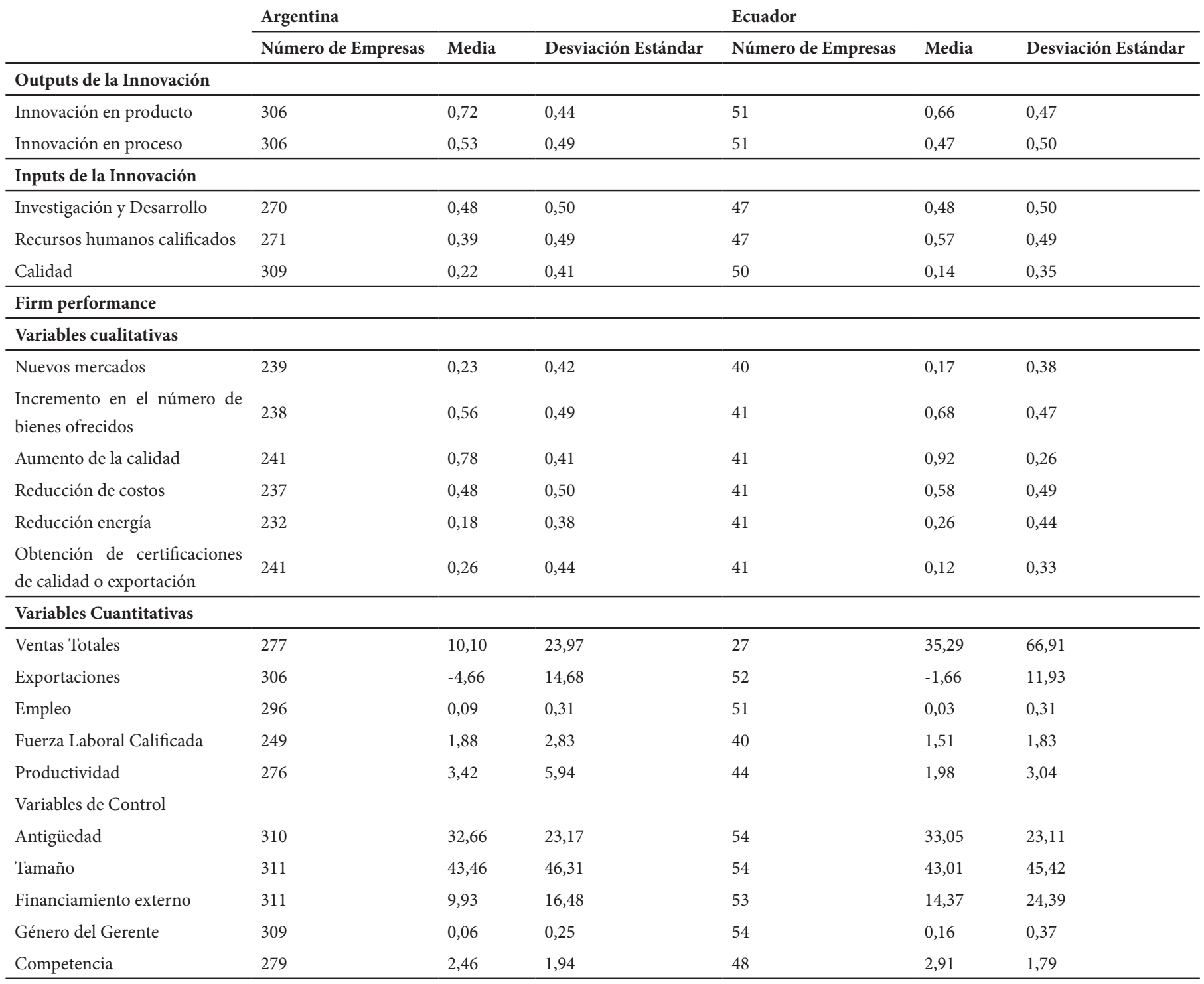

Fuente: Elaboración propia a partir de datos de la Enterprise Survey del Banco Mundial a empresas del sector manufacturero en la Argentina y en el Ecuador, Panel (2006-2010). 
Anexo No. 2

Codificación de variables cualitativas y cuantitativas de los efectos de la innovación del estudio en el cuestionario de la Enterprise Survey

\begin{tabular}{l|l}
\hline Variables Cualitativas & Código de la Variable \\
\hline Incremento del número de bienes o servicios ofrecidos por el establecimiento & LACp16a \\
\hline Apertura de nuevos mercados & LACp16b \\
\hline Mejora de la calidad de bienes o servicios & LACp16d \\
\hline Reducción de los costos unitarios de producción & LACp16e \\
\hline Reducción del consumo de energía (electricidad, gas, etc.) & LACp16f \\
\hline Obtención de certificados de calidad o de exportación & LACp16g \\
\hline Variables Cualitativas & Código de la Variable \\
\hline Empleados a tiempo completo permanentes del último año 2010 & 11 \\
\hline Empleados a tiempo completo permanentes en el 2007 & 12 \\
\hline Ventas anuales del establecimiento en el año fiscal & $\mathrm{d} 2$ \\
\hline Exportaciones directas e indirectas & $\mathrm{d} 3 \mathrm{~b}, \mathrm{~d} 3 \mathrm{c}$ \\
\hline Trabajadores a tiempo completo especializados al final del año 2010 & $14 \mathrm{a}$ \\
\hline Trabajadores a tiempo completo no especializados en el último año & $14 \mathrm{~b}$ \\
\hline Productividad & $\mathrm{d} 2,11$ \\
\hline
\end{tabular}

Elaboración propia con base en Enterprise Survey del Banco Mundial (2006-2010). 